\title{
Catalytic Isomerization of Quadricyclane Using Fourier Transform Near-Infrared Absorption Spectroscopy: Diffusion, Conversion, and Temperature Effect
}

\author{
Hsiu-Fang Fan, ${ }^{\dagger}$ Chia-Yu Chang, ${ }^{\dagger}$ Thou-Long Chin, ${ }^{\dagger}$ Tong-Ing Ho, ${ }^{\dagger}$ and King-Chuen Lin $*, \dagger$, \\ Department of Chemistry, National Taiwan University, Taipei 106, Taiwan, and Institute of Atomic and \\ Molecular Sciences, Academia Sinica, Taipei 106, Taiwan
}

Received: August 24, 2005

\begin{abstract}
By using Fourier transform near-infrared (NIR) absorption spectroscopy, the kinetic behaviors of quadricyclane isomerization, as catalyzed by anhydrous $\mathrm{CuSO}_{4}$ in chloroform mixture with and without agitation, are presented. Given the acquired NIR spectra, the concentration decay of quadricyclane with the reaction time is determined with the aid of partial least-squares analysis. When the mixture is not agitated, the diffusion coefficients in chloroform are evaluated to be $(3.8 \pm 0.1) \times 10^{-5} \mathrm{~cm}^{2} \mathrm{~s}^{-1}$ at $27^{\circ} \mathrm{C}$ and $(4.4 \pm 0.1) \times 10^{-5}$ $\mathrm{cm}^{2} \mathrm{~s}^{-1}$ at $39^{\circ} \mathrm{C}$. In the size-dependent experiments of the catalyst, the one-site and two-site coordinated conversion rate constants are further determined to be $(8.5 \pm 5.9) \times 10^{-6} \mathrm{~s}^{-1} A^{-1}$ and $(2.2 \pm 0.8) \times 10^{-8} \mathrm{~s}^{-1}$ $A^{-2}$, respectively, at $27^{\circ} \mathrm{C}$ and $(1.3 \pm 0.8) \times 10^{-5} \mathrm{~s}^{-1} A^{-1}$ and $(1.92 \pm 0.01) \times 10^{-6} \mathrm{~s}^{-1} A^{-2}$, respectively, at $39^{\circ} \mathrm{C}$. $A$ denotes the total catalyst surface area per unit effective volume of solvent. Accordingly, the activation energies for one-site and two-site coordination are evaluated to be 24.8 and $286.2 \mathrm{~kJ} \mathrm{~mol}^{-1}$, respectively. The reaction is dominated by one-site coordination (1:1 complex) between the reactant and the catalyst. Unless temperature increases, the two-site coordinated reaction may be ignored. In contrast, when analogous experiments are performed in the stirred solution, the diffusion factor is ignored but the conversion rate constants rise due to the increase of collision frequency. For instance, the one-site and two-site coordinated rate constants are increased to $(1.7 \pm 1.4) \times 10^{-5} \mathrm{~s}^{-1} A^{-1}$ and $(1.27 \pm 0.06) \times 10^{-5} \mathrm{~s}^{-1} A^{-2}$ at $39^{\circ} \mathrm{C}$. The two-site coordinated reaction rate is enhanced by a factor of 10 . Thus, isomerization may proceed via both 1:1 and 1:2 coordination between the reactant and the catalyst. The Arrhenius plot yields the corresponding activation energies to be $24 \pm 3$ and $275 \pm 3 \mathrm{~kJ} \mathrm{~mol}^{-1}$. The activation energies remain constant, no matter whether the solution is agitated or not.
\end{abstract}

\section{Introduction}

Interconversion of quadricyclane and norbornadiene has attracted considerable attention because of its potential for use in solar energy storage, ${ }^{1,2}$ molecular switches, ${ }^{3,4}$ optical waveguides, ${ }^{5}$ and photochromic chemsensors. ${ }^{6}$ In the application of solar energy storage, energy can be stored when norbornadiene is photochemically converted to quadricyclane and then released when quadricyclane is catalytically converted to norbornadiene. The quadricyclane-norbornadiene system is advantageous in several aspects. The conversion enthalpy is as large as $89 \mathrm{~kJ} \mathrm{~mol}^{-1}$, showing more energy that may be stored than most other candidates. Quadricyclane with an activation energy of $141 \mathrm{~kJ} \mathrm{~mol}^{-1}$ may last up to $140 \mathrm{~h}$ at $100{ }^{\circ} \mathrm{C},{ }^{7-9}$ thereby the energy can be saved for a long period. Nevertheless, norbornadiene cannot absorb solar light $>300 \mathrm{~nm}$; the conversion efficiency becomes as low as $\sim 0.05$ without the use of a photosensitizer. ${ }^{10,11}$ For this reason, its derivatives have been developed in the hope of efficiently absorbing the light waves longer than $300 \mathrm{~nm}$ and achieving better conversion efficiency without the aid of a photosensitizer. Thus far, such investigation has not yet been successful. Although the absorption wavelength has been made to red shift, the energy storage efficiency turns out to be worse than in the original system.

* To whom correspondence should be addressed. Fax: 886-2-23621483. E-mail: kclin@ccms.ntu.edu.tw.

$\dagger$ National Taiwan University.

$\doteqdot$ Academia Sinica.
Among those potential catalysts adopted in heterogeneous complex reactions, ${ }^{12-20}$ the $\mathrm{Sn}$ (II) and $\mathrm{Cu}$ (II) salts are frequently used for investigating the kinetics of the conversion of quadricyclane to norbornadiene. In the catalytic conversion reaction, Moore and co-workers determined the quadricyclane and norbornadiene concentrations by gas chromatography. ${ }^{13}$ Their procedure requires the removal and analysis of aliquots of the reaction mixture at intervals during the reaction. To develop an in situ monitoring of the concentration variation for both substances, Vickers and co-workers demonstrated with fiberoptic Raman spectroscopy the potential for automated, nondestructive, and continuous probing of the isomerization. ${ }^{14} \mathrm{Nev}$ ertheless, the suspended solids increased the sample opacity. As an alternative method, we developed Fourier transform nearinfrared (NIR) absorption spectroscopy to investigate the catalytic reactions. ${ }^{15,16}$ The reaction mixture was not agitated so as to avoid the effect of sample heterogeneity. Given the NIR absorption spectra acquired, the concentrations of reactant and product can be simultaneously determined with the analysis of partial least-squares (PLS). ${ }^{21-23}$

Moore and co-workers suggested that both one-site and twosite coordination of quadricyclane, with rate constants $2.9 \times$ $10^{-2} \mathrm{~min}^{-1} \mathrm{~g}^{-1}$ and $7.3 \times 10^{-2} \mathrm{~min}^{-1} \mathrm{~g}^{-2}$, respectively, for the $\mathrm{CuSO}_{4}$ catalyst in benzene, may lead to the production of norbornadiene. ${ }^{13}$ In our previous work, ${ }^{16}$ if a one-site coordinated reaction was assumed to dominate, then our result of 2.7 $\times 10^{-2} \mathrm{~min}^{-1} \mathrm{~g}^{-1}$ was consistent with that obtained above in a 
stirred solution. ${ }^{13}$ Nevertheless, if the contribution of two-site coordination was included, our one-site coordinated rate constant became smaller. In another similar experiment with Raman spectroscopy, Vickers and co-workers obtained a consistent second-order depletion rate constant of $2.7 \times 10^{-2} \mathrm{~min}^{-1} \mathrm{~g}^{-1}$ for quadricyclane with $\mathrm{CuSO}_{4}$ as the catalyst, but they did not look into the reaction mechanism. ${ }^{14}$ Apparently, the kinetics of such catalysis has not been well understood yet, including whether a two-site coordinated complex may play a significant role in the surface-mediated reaction, how temperature affects the reaction, how the catalyst reduces the activation energy, and whether the stirring motion may change the kinetic behavior or the mechanism. This work attempts to clarify these problems.

Herein, the kinetic behaviors of quadricyclane isomerization are investigated simultaneously under the conditions of either a still or stirred mixture. When the mixture stands still, the kinetic event becomes more complicated. The additional diffusion phenomena have to be taken into account. Fourier transform NIR absorption spectroscopy is used to measure the $\mathrm{CuSO}_{4}$-catalyzed isomerization of quadricyclane in the supernatant chloroform solution. With the aid of a rate model, diffusion and isomerization of quadricyclane may be well characterized. The isomerization rate constants thus obtained will be compared with those acquired in a stirred mixture. Then, the temperature dependence of the catalytic reaction is analyzed for these two cases. The activation energies for one-site and two-site coordination may be determined accordingly.

\section{Experimental Section}

A step-scan Fourier transform spectrometer (FTS, IFS-66v/ $\mathrm{s}$, Bruker) equipped with a tungsten lamp as the NIR radiation source and a Si diode detector suitable for the wavelength range from 6000 to $25000 \mathrm{~cm}^{-1}$. The FTS can be operated in either step-scan or rapid-scan mode with time resolutions on the order of nanoseconds and milliseconds, respectively. ${ }^{15,16,24-26}$ The rapid-scan mode is selected for the rate determination of the current catalytic isomerization. The interferogram of each scan, completed within milliseconds, was Fourier transformed and displayed.

The catalytic reactions were conducted either in a still or in a stirred mixture. The procedures for the former experiments have been described previously. ${ }^{16}$ In brief, about $7 \mathrm{~g}$ of the catalyst $\mathrm{CuSO}_{4}$ (Merck) was added in a cuboid cell $(1 \mathrm{~cm} \times 5$ $\mathrm{cm} \times 4.8 \mathrm{~cm}$ ), followed by slow addition of the chloroform solvent. After the suspended salts had settled in the cell, the light beam restricted to a cross section of $2 \mathrm{~mm}$ in diameter was guided to propagate through the solvent layer $\sim 0-1 \mathrm{~mm}$ above the solid surface. The NIR spectrum of the solvent background was acquired and averaged for 30 scans. Then, aliquots of chloroform drawn from the solvent layer were well mixed with $15-30 \mu \mathrm{L}$ quadricyclane or norbornadiene and carefully returned to the sample cell. NIR spectra were recorded at 30-s intervals over a period of $4 \mathrm{~h}$. Each spectrum was an average of 10 measurements. For the experiment of temperature effect, a homemade minithermostat was applied to keep the cuboid cell at either 27 or $39{ }^{\circ} \mathrm{C}$ during the reaction.

In the alternative experiments, a glass bottle, filled with 10 $\mathrm{mL}$ of chloroform solvent and a small amount of catalyst, was stirred in a thermostat with a magnet bar. The reaction was initiated by addition of $70 \mu \mathrm{L}$ of quadricyclane to the mixture. After different reaction periods from 5 up to $45 \mathrm{~min}$, the solution layer was filtered to a sample cell, followed by acquisition of NIR spectra with FTS. The filtrates without the catalyst left inside may not cause any further reaction. The procedures were repeatedly performed with variation of the catalyst amount from 0.2 to $0.8 \mathrm{~g}$. The measurements of temperature dependence from 30 to $44{ }^{\circ} \mathrm{C}$, as controlled in the thermostat, were similarly conducted.

Quadricyclane and norbornadiene in chloroform exhibit the maximum NIR absorption at 8840 and $8900 \mathrm{~cm}^{-1}$, respectively, which overlap with the solvent spectra. Fortunately, when the solvent background spectrum is subtracted, only the band at $8700 \mathrm{~cm}^{-1}$ leaves minor noise in the baseline. ${ }^{16}$ The change of the absorption bands during the reaction is large enough to allow for determination of the individual concentrations of quadricyclane and norbornadiene with the aid of the PLS method. ${ }^{21-23}$ In this work, 480 sets of the NIR absorption spectra for each catalytic reaction system were acquired, while 56 calibration sets of various concentration combinations of quadricyclane and norbornadiene each in the range $0.005-0.100 \mathrm{M}(\mathrm{mol} / \mathrm{L})$ were prepared. The fit between the predicted and actual concentrations, which is characterized by a straight line having a slope of 1.00 and a regression coefficient $>0.98$, gives rise to the quadricyclane or norbornadiene concentrations in the reaction mixture.

For the measurements of the temperature effect in a still solution, the PLS analyses at different temperatures of 27 or $39{ }^{\circ} \mathrm{C}$ were applied to find the concentrations of the related substances during the reaction. In contrast, the filtrates collected from the stirred solution were cooled to $27^{\circ} \mathrm{C}$ before the NIR spectra were acquired. Thus, the PLS analysis only at $27{ }^{\circ} \mathrm{C}$ was performed for the reactions at varied temperatures.

The reactant quadricyclane (quadricyclo[2.2.1.0.0] heptane) was photosynthesized from norbornadiene (bicyclo[2.2.1] hepta2,5-diene) with acetonitrile as a photosensitizer. The light at $365 \mathrm{~nm}$ was irradiated for about $8 \mathrm{~h}$ /day for 4 weeks. Its purity $>98 \%$ was examined by ${ }^{1} \mathrm{H}$ NMR spectroscopy. Norbornadiene (99\%, Aldrich) was used without further purification. The catalyst $\mathrm{CuSO}_{4}$ was heated at $140{ }^{\circ} \mathrm{C}$ for $12 \mathrm{~h}$ before use. The particle sizes were distributed into five groups of 38-44, 53$63,74-88,106-125$, and 149-177 $\mu \mathrm{m}$ through various standard mesh frames. $\mathrm{CHCl}_{3}$ was dried over molecular sieves with a $4-\AA$ p pore size.

\section{Kinetic Model}

When the mixture is not agitated, quadricyclane must rely on diffusion onto the solid surface to result in norbornadiene. The kinetic rate equation including the contribution of diffusion and conversion is thus expressed as ${ }^{15}$

$$
\frac{\mathrm{d} C}{\mathrm{~d} t}=D \frac{\mathrm{d}^{2} C}{\mathrm{~d} x^{2}}-k C
$$

where $\mathrm{C}$ is the concentration of quadricyclane, $D$, its diffusion coefficient, and $x$, the depth measured from the top of the solution, defined as zero, to the bottom of the cell.

As reported previously, ${ }^{16}$ eq 1 can be solved numerically by a one-dimensional method using a program based on Matlab 6.5 in which input data such as boundary conditions, the height of solvent above the catalyst surface, and the effective depth of the catalyst stacks are required. The effective depth of the catalyst can be evaluated by measuring the additional height of the solvent layer when a given weight of catalyst is deposited in the cell. It reflects the solvent amount in the interstices of the solid stacks. Two parameters related to the diffusivity are required in the simulation. One represents the diffusion coefficient of quadricyclane in the solvent layer, which is fitted when $x$ is less than the solvent height, and the other one represents 


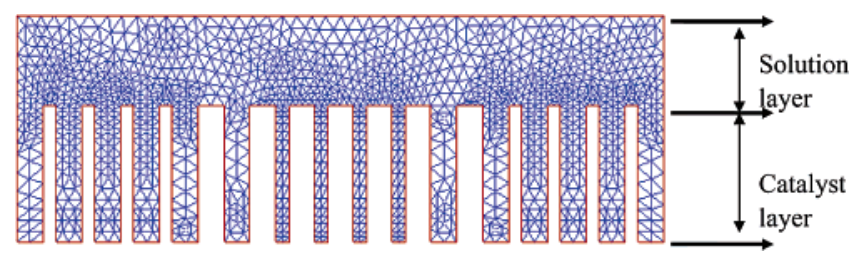

Figure 1. Model cell in which the solvent amount inside the solid layer is divided into numerous branches. The entire system is broken up into many tiny triangular unit cells. The concentrations of quadricyclane in all the triangular unit cells are solved as functions of time and space.

the diffusion coefficient in the solid stacks, which is fitted when $x$ is larger than the solvent height.

In this work, eq 1 with diffusion alone can be solved technically with a two-dimensional method. The solvent amount in the interstices of the solid stacks is distributed into numerous branches, as depicted in Figure 1. The ratio of the volumes occupied between the solvent and the solid particles in the entire branch region is subject to the experimental conditions. The numerical analysis of eq 1 is performed by a finite element method. That is, the whole system in Figure 1 is broken up into many tiny triangular unit cells, which may be spatially characterized with a two-dimensional coordinate. Given the boundary conditions, $\mathrm{d} C / \mathrm{d} x=0$, valid for all the edges of the model cell, the height of the solvent above the catalyst surface, and the volume ratio in the branch region, the concentrations of quadricyclane in all the triangular unit cells may be solved as functions of time and space, according to a written program based on the Matlab 6.5 environment. To compare with the experimental observation of the reactant decay, each time- and space-dependent simulated curve is integrated within the zone where the NIR probe beam propagates through the solution. Note that the diffusion coefficient of quadricyclane in the solvent is the only parameter to fit the obtained decay curve. The quality of simulation can be improved by increasing the number of branches, but it will not be improved further when the number exceeds eight. The optimized value remains a constant, irrespective of the size change of the catalyst.

Despite the improved accuracy of the diffusion coefficient evaluation, the developed two-dimensional method cannot handle the complicated event to determine conversion and diffusion simultaneously. Therefore, in this work, we first apply the two-dimensional method to find out the diffusion coefficient of quadricyclane in the solvent. Given this parameter, a onedimensional method is adopted to fit the geometry factor related to the solid phase. For one-dimensional diffusion, the diffusion of quadricyclane may be hindered while moving through the catalyst stacks along the $x$ direction, and thus, the fitted geometry factor is smaller than unity. In this manner, the diffusion coefficient of quadricyclane inside the solid stacks is evaluated by multiplication of the diffusion coefficient in the solvent and the geometry factor. After both the diffusion coefficients are obtained, the conversion rate constant is analogously fitted with the one-dimensional method. ${ }^{16}$

\section{Results and Discussion}

A. Determination of Kinetic Constants in Solution without Agitation. Since the norbornadiene product has a similar structure and properties as quadricyclane, it is used as a substitute to determine the diffusion coefficients for quadricyclane in the catalytic system. Its concentration as a function of time is evaluated by PLS analysis, as described in the Experimental Section. The measured concentration change of

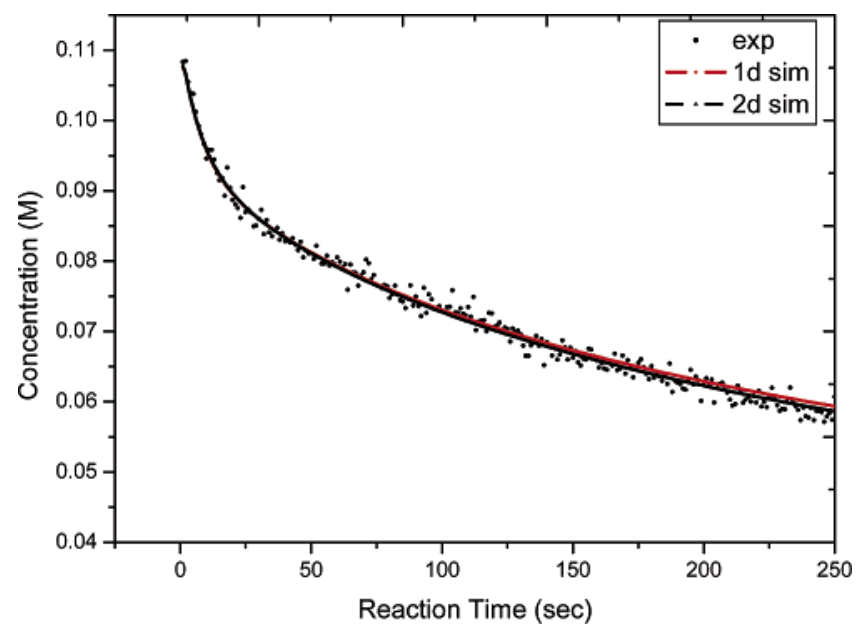

Figure 2. Time-dependent concentration of norbornadiene caused by diffusion in chloroform in the presence of $\mathrm{CuSO}_{4}$. The measurements with the symbol are conducted at the solution height of $0.45 \mathrm{~cm}$ and the catalyst weight of $7 \mathrm{~g}$. The corresponding fit with the onedimensional method yields $3.8 \times 10^{-5}$ and $1.29 \times 10^{-5} \mathrm{~cm}^{2} \mathrm{~s}^{-1}$ for the diffusion coefficients in the solvent and inside the solid phase, respectively. The fit with the two-dimensional method yields $3.8 \times$ $10^{-5} \mathrm{~cm}^{2} \mathrm{~s}^{-1}$ for the diffusion coefficient. The catalyst particle size is $38-44 \mu \mathrm{m}$.

TABLE 1: Particle-Size Dependence of Diffusion Coefficients in Solvent/Solid Phases and Pseudo-First-Order Conversion Rate Constants at $27^{\circ} \mathrm{C}$

\begin{tabular}{lccc}
\hline $\begin{array}{c}\text { catalyst } \\
\text { size }(\mu \mathrm{m})\end{array}$ & $\begin{array}{c}D_{1}{ }^{a} \\
\left(\times 10^{5} \mathrm{~cm}^{2} \mathrm{~s}^{-1}\right)\end{array}$ & $\begin{array}{c}D_{2}{ }^{b} \\
\left(\times 10^{6} \mathrm{~cm}^{2} \mathrm{~s}^{-1}\right)\end{array}$ & $\begin{array}{c}k_{\mathrm{I}}^{c} \\
\left(\times 10^{3} \mathrm{~s}^{-1}\right)\end{array}$ \\
\hline $149-177$ & 3.8 & 1.43 & 2.2 \\
$106-125$ & 3.8 & 1.33 & 3.4 \\
$74-88$ & 3.8 & 1.29 & 5.0 \\
$53-63$ & 3.8 & 1.22 & 8.2 \\
$38-44$ & 3.8 & 1.29 & 14.5
\end{tabular}

${ }^{a}$ The term $D_{1}$ denotes the diffusion coefficient in chloroform. ${ }^{b}$ The term $D_{2}$ denotes the diffusion coefficient in the catalyst. ${ }^{c}$ The term $k_{\mathrm{I}}$ denotes the pseudo-first-order catalytic rate constant.

norbornadiene due to diffusion is plotted in Figure 2. The observed data are simulated with either one-dimensional or twodimensional methods to determine the diffusivity. The resultant diffusion coefficients in the solvent are consistent. We alternatively use $\mathrm{NaCl}$ salt to replace $\mathrm{CuSO}_{4}$, since the former salt cannot catalyze quadricyclane. The determined diffusion coefficient of quadricyclane in $\mathrm{CHCl}_{3}$ also agrees with those found using norbornadiene as a substitute. The determined diffusion coefficients remain consistent, irrespective of variation of the solution volume. As the particle size of $\mathrm{CuSO}_{4}$ is changed following the distributions of $38-44,53-63,74-88,106-$ 125 , and $149-177 \mu \mathrm{m}$, the fits of the diffusion coefficients are similarly carried out. The results are listed in Table 1 . The diffusion coefficients in the solvent layer are determined to be $(3.8 \pm 0.1) \times 10^{-5} \mathrm{~cm}^{2} \mathrm{~s}^{-1}$ at $27^{\circ} \mathrm{C}$, while the one inside the $\mathrm{CuSO}_{4}$ catalyst decreases normally with a decrease in the particle size. The diffusion tends to be slower, since the catalyst stack becomes more tightly packed as a result of the decreased size. With the one-dimensional method, the size effect can be characterized by a geometry factor, which becomes smaller when the solid pack is tighter. For instance, it is found to be 0.34 and 0.38 for $\mathrm{CuSO}_{4}$ with the size distributions of 74-88 and $149-177 \mu \mathrm{m}$, respectively. In contrast, in terms of the twodimensional method with the branch model, a single parameter of diffusivity can be fitted to all the concentration decay curves acquired with different particle sizes. The obtained diffusion coefficients, which remain independent of the size variation, 


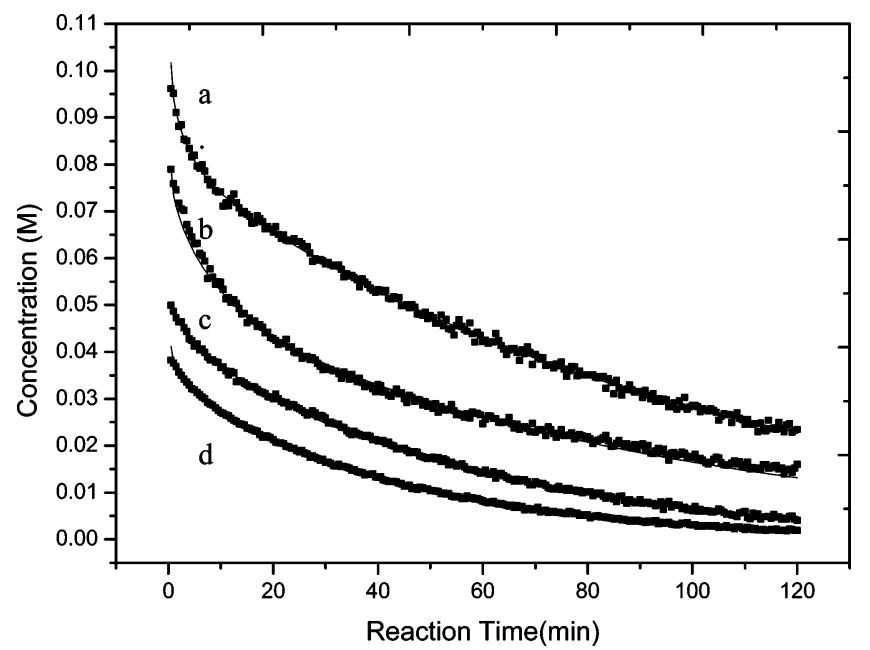

Figure 3. Reaction-time dependence of the concentration of quadricyclane catalyzed by $7 \mathrm{~g}$ of $\mathrm{CuSO}_{4}$ in chloroform at $27{ }^{\circ} \mathrm{C}$ with solution heights of (a) 0.4 , (b) 0.55 , (c) 0.45 , and (d) $0.35 \mathrm{~cm}$. The solid lines indicate the corresponding simulated curves with the diffusion coefficients fixed at $3.8 \times 10^{-5}$ and $1.43 \times 10^{-5} \mathrm{~cm}^{2} \mathrm{~s}^{-1}$ in the solution layer and the solid stacks, respectively. The fit yields the depletion rate constant of quadricyclane.

are in agreement with the one in the solvent phase using the one-dimensional method.

Because of hindrance of the flow along the vertical direction, the diffusion inside the solid stacks is found to be slower as simulated with the one-dimensional method. In contrast, for the two-dimensional method, the flow along horizontal and vertical directions are both taken into account such that the diffusion does not make any significant difference between the solvent and solid phases, if the interstices of the solid stacks are large enough like $\mathrm{CuSO}_{4}$. Furthermore, the branch model has removed the steric hindrance caused by particle shape and stacking. Unless the ratio of the volumes occupied by the solvent to the solid stacks is too small, a single parameter of diffusivity is appropriate enough to fit the observed concentration decay.

Given the diffusion coefficients in the two phases, the isomerization rate constant of quadricyclane catalyzed by $\mathrm{CuSO}_{4}$ can be evaluated by simulating the concentration decay. When the solvent amounts are varied, the obtained rate constants are consistent in the simulation. An example for the fit with the size distribution 149-177 $\mu \mathrm{m}$ under the conditions of different solvent amounts is shown in Figure 3, in which the reaction rate constant is the only parameter to adjust. The conversion rate constants are analogously evaluated as functions of the particle size of the catalyst. The results are summarized in Table 1. The catalytic conversion becomes faster with decreasing the particle size, since the total surfaces contacted by the reactant may increase. For instance, the pseudo-first-order conversion rate constants are found to be $(1.5 \pm 0.1) \times 10^{-2}$ and $(3.4 \pm$ $0.1) \times 10^{-3} \mathrm{~s}^{-1}$ with the size distributions of $38-44$ and $106-$ $125 \mu \mathrm{m}$, respectively.

The obtained pseudo-first-order rate constant, $k_{\mathrm{tot}}$, may be expressed as a sum of the one-site and two-site coordinated depletion rate constants, ${ }^{16}$

$$
k_{\mathrm{tot}}=k_{1} \frac{\Delta A}{\Delta V_{\mathrm{e}}}+k_{2}\left(\frac{\Delta A}{\Delta V_{\mathrm{e}}}\right)^{2}=k_{1} \frac{6 W}{d a \Delta V_{\mathrm{e}}}+k_{2}\left(\frac{6 W}{d a \Delta V_{\mathrm{e}}}\right)^{2}
$$

where $\Delta A$ is the total surface area of the solid, $\Delta V_{\mathrm{e}}$, the effective volume of solvent squeezed in the catalyst stacks, $W$, the weight of catalyst, $d$, the density of catalyst, and $a$, the average diameter of the spherical particles. The effective volume of solvent inside

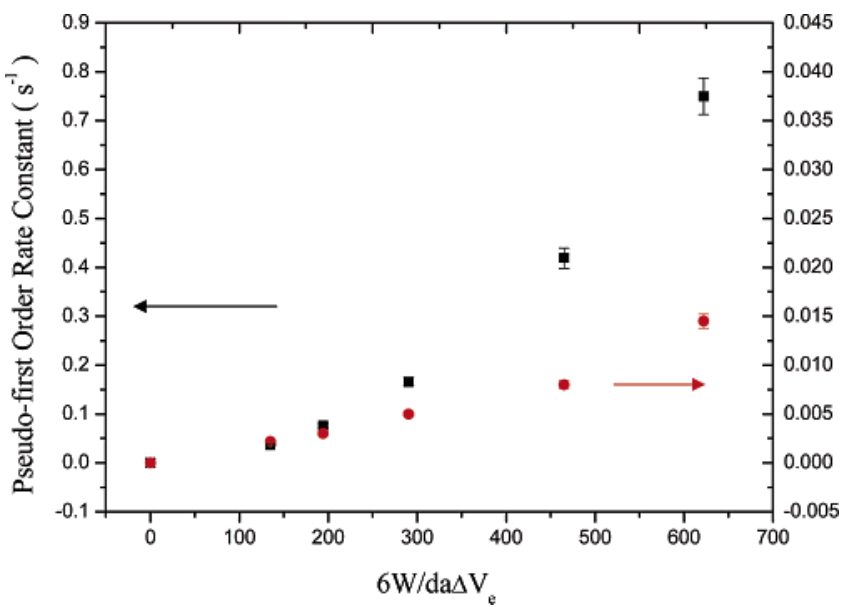

Figure 4. Particle-size dependence of the pseudo-first-order rate constant, $k_{\text {tot }}$, to yield one-site and two-site coordinated rate constants at $27^{\circ} \mathrm{C}$ (denoted by ) and $39^{\circ} \mathrm{C}$ (denoted by $\mathbf{0}$ ). The catalyst weight, $W$, and the density, $d$, are fixed at $7 \mathrm{~g}$ and $3.6 \mathrm{~g} \mathrm{~cm}^{-3}$, but the effective volume, $\Delta V_{\mathrm{e}}$, is subject to the particle size and measured for each data point.

the catalyst stacks is readily evaluated from the bottom-side area of the cell multiplied by the additional height of the solvent layer when the catalyst is added. Given the evaluated effective solvent volume, the weight of catalyst, and the density $3.6 \mathrm{~g} / \mathrm{cm}^{3}$ of anhydrous $\mathrm{CuSO}_{4}$, as shown in Figure 4, the plot of $k_{\text {tot }}$ versus $6 W / d a \Delta V_{\mathrm{e}}$ yields one-site and two-site coordinated rate constants, $k_{1}$ and $k_{2}$, to be $(8.5 \pm 5.9) \times 10^{-6} \mathrm{~s}^{-1} A^{-1}$ and $(2.2 \pm$ $0.8) \times 10^{-8} \mathrm{~s}^{-1} \mathrm{~A}^{-2}$, respectively, at $27^{\circ} \mathrm{C}$. $A$ denotes the total catalyst surface area per unit effective volume of solvent. The rate $k_{1}$ is slightly smaller than the previous value $1.3 \times 10^{-5}$ $\mathrm{s}^{-1} A^{-1}$ as calculated with a single average diameter, but the value thus obtained in this work is more reliable. The rate $k_{2}$ is found to be 2 orders of magnitude smaller than $k_{1}$, suggesting that the two-site coordinated reaction should be negligible at the low temperature of $27^{\circ} \mathrm{C}$.

In a similar experiment, Moore and co-workers have obtained the one-site and two-site coordinated rate constants to be $4.8 \times$ $10^{-4} \mathrm{~s}^{-1} \mathrm{~g}^{-1}$ and $1.2 \times 10^{-3} \mathrm{~s}^{-1} \mathrm{~g}^{-2}$, respectively, for the $\mathrm{CuSO}_{4}$ catalyst in benzene by detection with GC-MS. Given, in eq 2, the particle diameter of $3.3 \times 10^{-3} \mathrm{~cm}$ and a total volume of $10 \mathrm{~mL}$ in a stirred solution to substitute for the effective solvent volume, ${ }^{13}$ then their conditions may lead to the corresponding values of $9.6 \times 10^{-6} \mathrm{~s}^{-1} A^{-1}$ and $4.7 \times 10^{-7} \mathrm{~s}^{-1} A^{-2}$, in comparison with our results $(8.5 \pm 5.9) \times 10^{-6} \mathrm{~s}^{-1} A^{-1}$ and $(2.2 \pm 0.8) \times 10^{-8} \mathrm{~s}^{-1} A^{-2}$ at $27^{\circ} \mathrm{C}$. The one-site rate constants are consistent with each other, but the two-site rate constants differ by 20 times.

B. Temperature Dependence in Still Solution. To evaluate how the activation energy of $141 \mathrm{~kJ} \mathrm{~mol}^{-1}$ is reduced with the added $\mathrm{CuSO}_{4}$ catalyst, ${ }^{8,9}$ we move further to find out the diffusion and conversion of quadricyclane at the temperature of $39^{\circ} \mathrm{C}$. The PLS analysis is again applied at this temperature to give rise to the concentration decay of quadricyclane during the reaction. All the experimental processes are otherwise identical to those conducted at $27{ }^{\circ} \mathrm{C}$. Figure 5 shows an example for observation and simulation of the concentration decay catalyzed with the size distribution $74-88 \mu \mathrm{m}$ of $\mathrm{CuSO}_{4}$. The diffusivity and conversion rate constants are analogously evaluated as functions of the particle size of the catalyst. The results are summarized in Table 2 . The diffusion coefficient in chloroform increases to $(4.4 \pm 0.1) \times 10^{-5} \mathrm{~cm}^{2} \mathrm{~s}^{-1}$ at $39^{\circ} \mathrm{C}$. It is reasonable to find an increase of diffusivity with temperature. According to Stokes-Einstein equation, the diffusion 

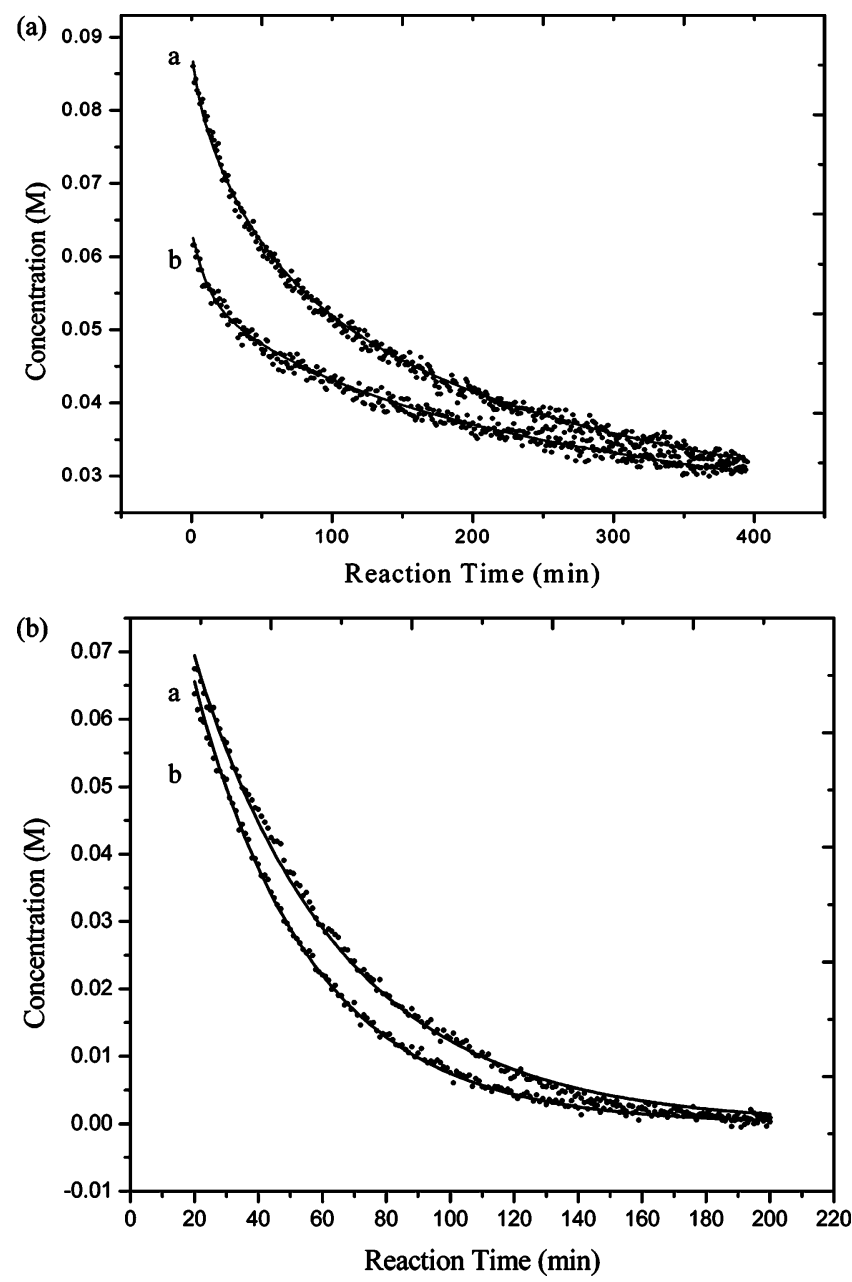

Figure 5. (a) Time-dependent concentration of norbornadiene caused by diffusion in chloroform in the presence of $7 \mathrm{~g}$ of $\mathrm{CuSO}_{4}$ at $39{ }^{\circ} \mathrm{C}$ with solution heights of (a) 0.41 and (b) $0.35 \mathrm{~cm}$. The solid lines indicate the corresponding simulated curves to yield the diffusion coefficients. (b) Reaction-time dependence of the concentration of quadricyclane catalyzed by $7 \mathrm{~g}$ of $\mathrm{CuSO}_{4}$ in chloroform at $39^{\circ} \mathrm{C}$ with solution heights of (a) 0.36 and (b) $0.32 \mathrm{~cm}$. The solid lines indicate the corresponding simulated curves with the diffusion coefficients fixed at $4.4 \times 10^{-5}$ and $1.77 \times 10^{-5} \mathrm{~cm}^{2} \mathrm{~s}^{-1}$ in the solution layer and the solid stacks, respectively. The fit yields the depletion rate constant of quadricyclane.

TABLE 2: Particle-Size Dependence of Diffusion Coefficients in Solvent/Solid Phases and Pseudo-First-Order Conversion Rate Constants at $39{ }^{\circ} \mathrm{C}$

\begin{tabular}{lccc}
\hline $\begin{array}{c}\text { catalyst } \\
\text { size }(\mu \mathrm{m})\end{array}$ & $\begin{array}{c}D_{1}{ }^{a} \\
\left(\times 10^{5} \mathrm{~cm}^{2} \mathrm{~s}^{-1}\right)\end{array}$ & $\begin{array}{c}D_{2}{ }^{b} \\
\left(\times 10^{6} \mathrm{~cm}^{2} \mathrm{~s}^{-1}\right)\end{array}$ & $\begin{array}{c}k_{\mathrm{I}}^{c} \\
\left(\times 10^{3} \mathrm{~s}^{-1}\right)\end{array}$ \\
\hline $149-177$ & 4.4 & 1.77 & 0.0365 \\
$106-125$ & 4.4 & 1.77 & 0.076 \\
$74-88$ & 4.4 & 1.77 & 0.166 \\
$53-63$ & 4.4 & 1.77 & 0.419 \\
$38-44$ & 4.4 & 1.77 & 0.75
\end{tabular}

${ }^{a}$ The term $D_{1}$ denotes the diffusion coefficient in chloroform. ${ }^{b}$ The term $D_{2}$ denotes the diffusion coefficient in the catalyst. ${ }^{c}$ The term $k_{\mathrm{I}}$ denotes the pseudo-first-order catalytic rate constant.

coefficient in solution is proportional to the term $T / \eta ; T$ and $\eta$ denote the absolute temperature and viscosity, respectively. The term $\eta$ can be replaced by $\exp \left(E_{\mathrm{a}} / R T\right)$, where $E_{\mathrm{a}}$ is the activation energy for quadricyclane motion in the chloroform solvent and $R$ is the gas constant. Given the values $E_{\mathrm{a}}=10 \mathrm{~kJ} \mathrm{~mol}^{-1}$ and $D_{1}=3.8 \times 10^{-5} \mathrm{~cm}^{2} \mathrm{~s}^{-1}$ at $27^{\circ} \mathrm{C}, D_{1}$ in chloroform at $39^{\circ} \mathrm{C}$ may be estimated to be $4.6 \times 10^{-5} \mathrm{~cm}^{2} \mathrm{~s}^{-1}$, consistent with the obtained value.
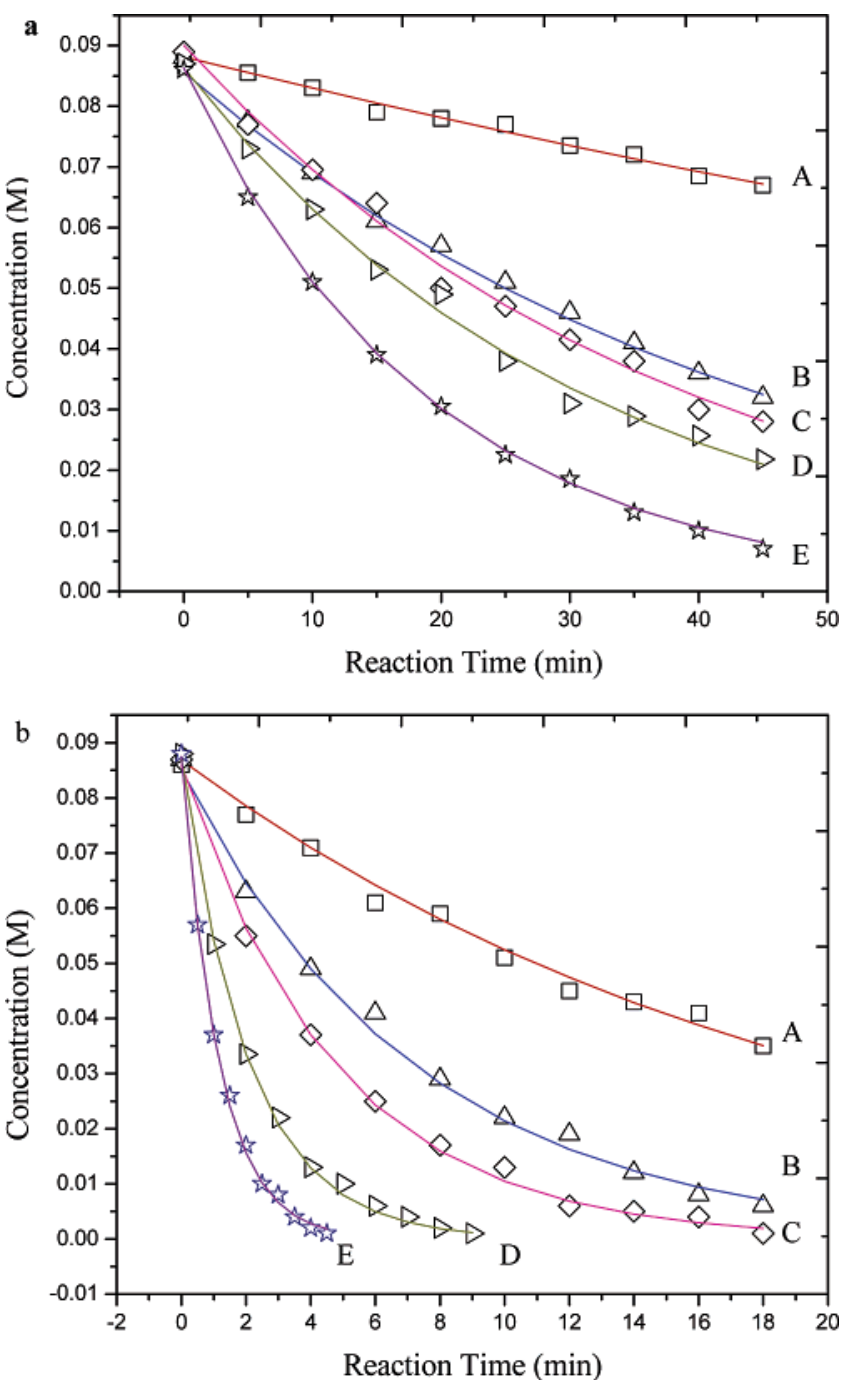

Figure 6. Concentration decay of quadricyclane, catalyzed by $\mathrm{CuSO}_{4}$ in the stirred chloroform mixture, as a function of the catalyst amounts at temperatures of (a) $30{ }^{\circ} \mathrm{C}(\mathrm{A} 0.2 ; \mathrm{B} 0.4 ; \mathrm{C} 0.5$; D 0.6 ; E $0.8 \mathrm{~g})$ and (b) $39^{\circ} \mathrm{C}$ (A 0.2; B 0.3; C 0.4; D 0.6; E $0.8 \mathrm{~g}$ ). The decay curves are fitted using an exponential function to yield the corresponding conversion rate constants.

TABLE 3: One-Site and Two-Site Coordinated Conversion Rate Constants in the Stirred Mixture at Different Temperatures

\begin{tabular}{ccc}
\hline & $k_{1}\left(\times 10^{5} \mathrm{~s}^{-1} A^{-1}\right)$ & $k_{2}\left(\times 10^{6} \mathrm{~s}^{-1} A^{-2}\right)$ \\
\hline $30^{\circ} \mathrm{C}$ & $1.2 \pm 0.5$ & $0.5 \pm 1.5$ \\
$35^{\circ} \mathrm{C}$ & $1.4 \pm 1.0$ & $2.3 \pm 0.3$ \\
$39^{\circ} \mathrm{C}$ & $1.7 \pm 1.4$ & $12.7 \pm 0.6$ \\
$44^{\circ} \mathrm{C}$ & $2.0 \pm 1.5$ & $89.5 \pm 6.7$
\end{tabular}

At high temperatures, the diffusivity inside the solid stacks is found to be almost independent of the particle size, showing different behavior from those observed at low temperatures (Tables 1 and 2). The difference of the steric effect due to the size distribution might be overlooked by the increase of thermal motion at high temperatures. But a detailed interpretation has not been known yet. Again, the one-site and two-site coordinated rate constants are determined from the plot of $k_{\text {tot }}$ versus $6 \mathrm{~W} /$ $d a \Delta V_{\mathrm{e}}$, as shown in Figure 4, yielding $k_{1}$ and $k_{2}$ to be $(1.3 \pm$ $0.8) \times 10^{-5} \mathrm{~s}^{-1} A^{-1}$ and $(1.92 \pm 0.01) \times 10^{-6} \mathrm{~s}^{-1} A^{-2}$. As the temperature increases, $k_{1}$ is enhanced by less than a factor of 2 but $k_{2}$ is remarkably enhanced by 2 orders of magnitude. Given the rate constants obtained at different temperatures, an Arrhenius equation is adopted to determine the one-site and two-site 

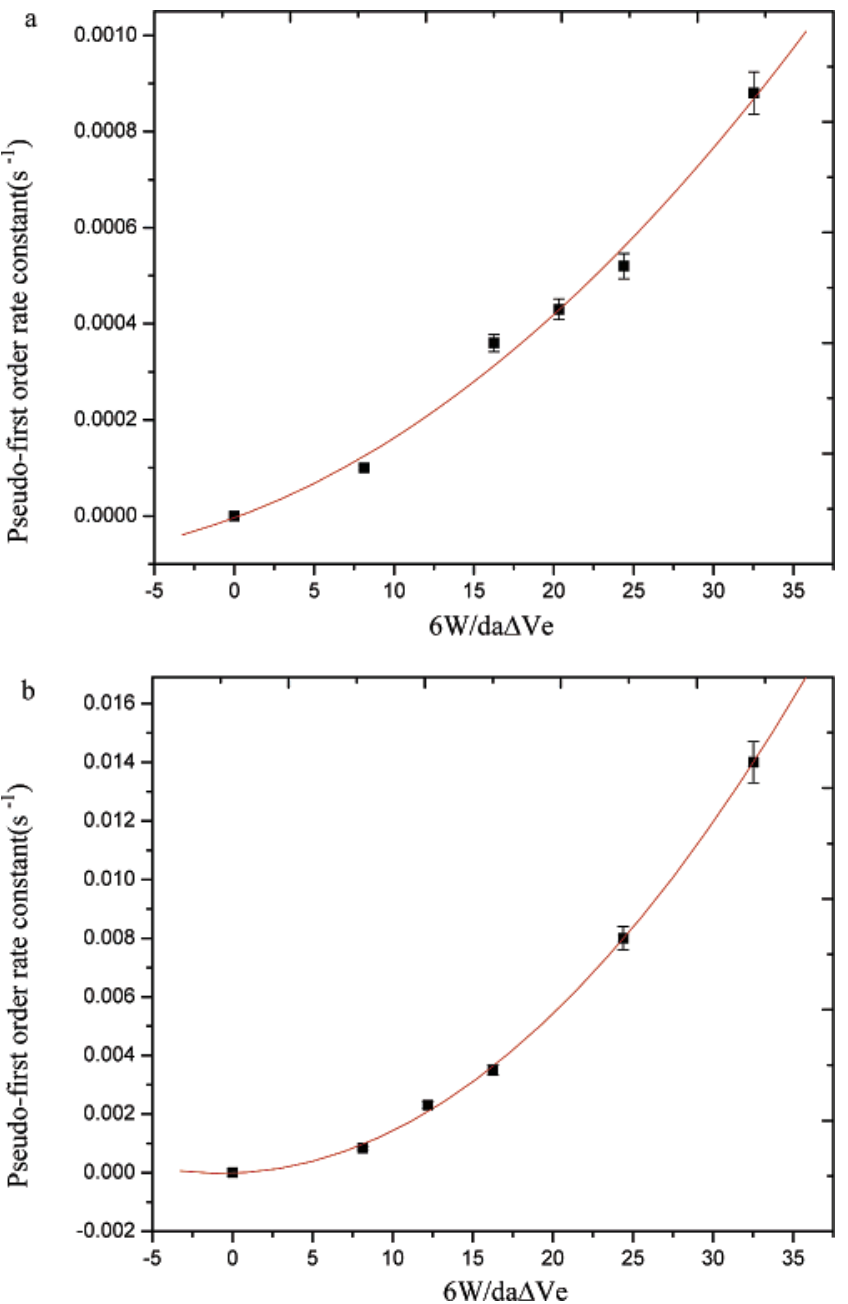

Figure 7. Plot of pseudo-first-order conversion rate constants as functions of $6 \mathrm{~W} / \mathrm{da} \Delta V_{\mathrm{e}}$ to yield one-site and two-site coordinated rate constants in the stirred mixtures at temperatures of (a) 30 and (b) 39 ${ }^{\circ} \mathrm{C}$. $W, d$, and $a$ denote the weight, density, and particle diameter of the catalyst, respectively, and $\Delta V_{\mathrm{e}}$ is the effective volume of the solution, which is replaced by a total volume of solution fixed at 10 $\mathrm{mL}$.

coordinated activation energies to be 24.8 and $286.2 \mathrm{~kJ} \mathrm{~mol}^{-1}$, respectively.

It is now possible, for the first time, to yield the activation energy for the quadricyclane isomerization catalyzed by $\mathrm{CuSO}_{4}$. This catalyst may facilitate the reaction by reducing markedly the activation energy. For the still solution, the reaction is primarily controlled via a 1:1 coordination between the reactant and the catalyst. The two-site coordinated reaction gives no appreciable contribution at low temperatures, such as $27^{\circ} \mathrm{C}$, but cannot be neglected as temperature increases.

C. Kinetic Rate Constants in Stirred Solution. In the experiments of stirred solution, the diffusion factor is ignored. We fixed the particle size of the catalyst at $38-44 \mu \mathrm{m}$ throughout the experiments. The concentrations of quadricyclane left in the filtrates as a function of the reaction time are analogously determined by the PLS analysis. As shown in Figure 6 , the concentration of quadricyclane decreases with the reaction time. The decay rate increases as a result of the increased amount of catalyst. Each decay curve may be fitted with an exponential function, as derived from eq 1 without the diffusion term involved. The conversion rate constants as a function of the catalyst amount may thus be evaluated. Similar experiments are carried out at varied temperatures of $30,35,39$, and $44{ }^{\circ} \mathrm{C}$. In
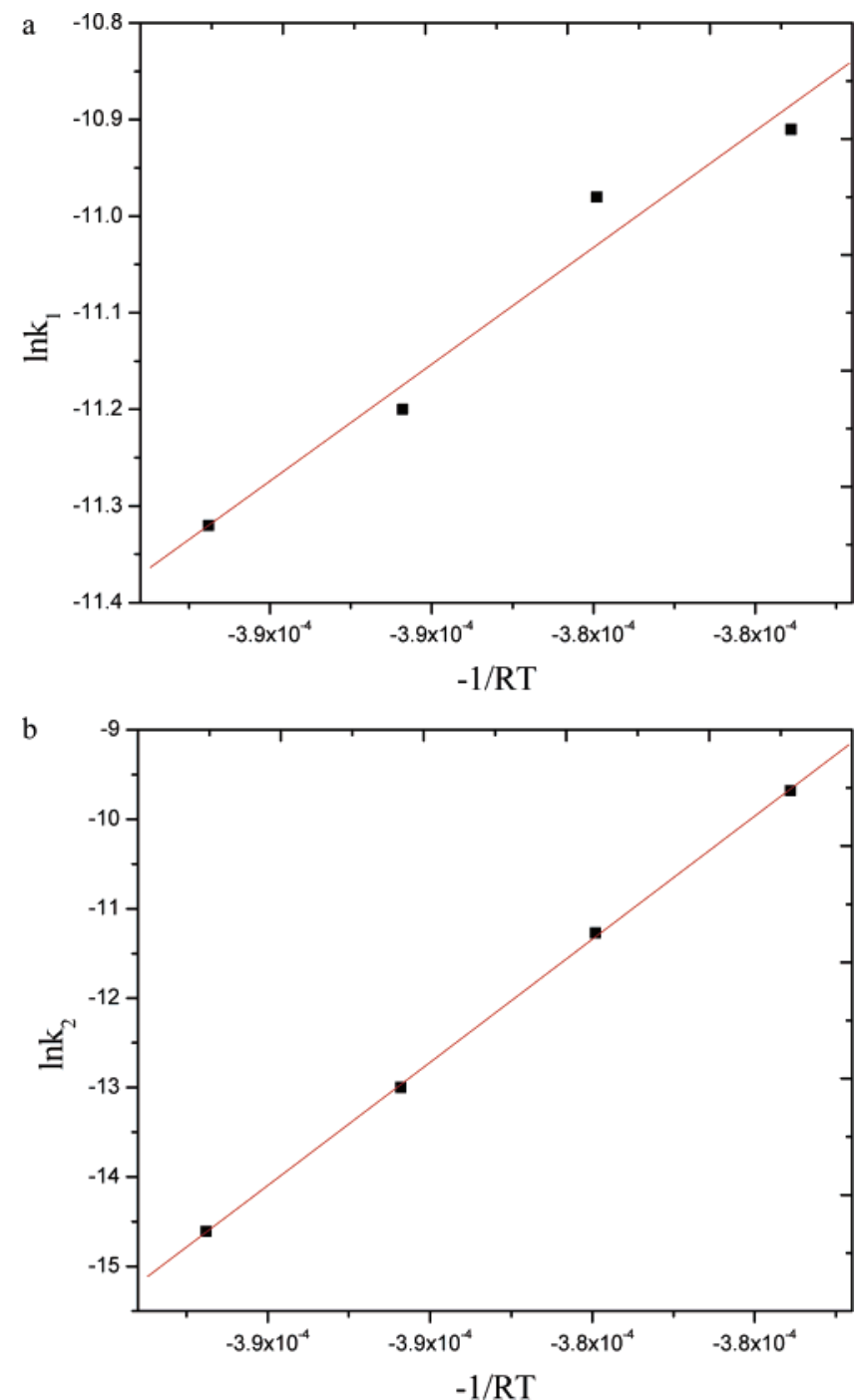

Figure 8. Arrhenius plots of one-site and two-site coordinated conversion rate constants, $k_{1}$ and $k_{2}$, against the reciprocal of temperature on the logarithmic scale. The slopes yield the corresponding activation energies.

this manner, the pseudo-first-order rate constants, $k_{\text {tot }}$, at different temperatures may be evaluated. According to eq 2, the plot of $k_{\text {tot }}$ versus $6 W / d a \Delta V_{\mathrm{e}}$ yields one-site and two-site coordinated rate constants, $k_{1}$ and $k_{2}$. The results are listed in Table 3 . The examples of the fit at 30 and $39{ }^{\circ} \mathrm{C}$ are shown in Figure 7. Herein, the effective solvent volume, $\Delta \mathrm{V}_{\mathrm{e}}$, in eq 2 is replaced by the total solvent volume, as fixed at $10 \mathrm{~mL}$ throughout the experiments. Given the kinetic data, $k_{1}$ and $k_{2}$, in Table 3, as shown in Figure 8, the Arrhenius plot yields the corresponding activation energy to be $24 \pm 3$ and $275 \pm 3 \mathrm{~kJ} / \mathrm{mol}$.

The obtained activation energies for one-site and two-site coordinated reactions are consistent with those obtained in a still solution. According to the Arrhenius equation, the activation energy should remain constant, no matter whether the solution is agitated. Nevertheless, the collision frequency is enhanced under the stirred condition. While inspecting the kinetic data at $39{ }^{\circ} \mathrm{C}$, we find that $k_{1}$ and $k_{2}$ are $(1.3 \pm 0.8) \times 10^{-5} \mathrm{~s}^{-1} A^{-1}$ and $(1.92 \pm 0.01) \times 10^{-6} \mathrm{~s}^{-1} A^{-2}$, respectively, in the still solution, but the values increase to $(1.7 \pm 1.4) \times 10^{-5} \mathrm{~s}^{-1} A^{-1}$ and $(1.27 \pm 0.06) \times 10^{-5} \mathrm{~s}^{-1} A^{-2}$ in the stirred solution. The isomerization rate constants become larger in the stirred solution, since the collision frequencies increase. This trend is consistent with the report by Moore and co-workers. ${ }^{13}$ Especially for the 
two-site coordinated reaction, agitation enhances the collision probabilities for a 1:2 coordination and provides additional kinetic energy to overcome the activation barrier. Thus, the $k_{2}$ value is enhanced by 1 order of magnitude in the temperature rage from 27 to $39^{\circ} \mathrm{C}$ studied.

\section{Conclusion}

We have investigated the kinetics of heterogeneous catalysis in either a still or a stirred mixture. By using Fourier transform NIR absorption spectroscopy combined with a simulation model, the kinetic parameters including the diffusion coefficients in the liquid/solid phases, conversion rate constants, and activation energies are evaluated for isomerization of quadricyclane catalyzed by anhydrous $\mathrm{CuSO}_{4}$. The acquired NIR spectra are analyzed with the PLS method to resolve quadricyclane and norbornadiene concentrations during the reaction. When the heterogeneous mixture is not agitated, the further experiments of particle size dependence yield the one-site and two-site coordinated rate constants. The one-site coordinated conversion rate constants are close to those obtained in the stirred solution, but the two-site coordinated rate constants are smaller by 1 order of magnitude. This fact rises from an enhancement of the collision frequency in the stirred solution. If the solution is not agitated, the isomerization is dominated by $1: 1$ coordination between quadricyclane and the catalyst and the contribution of two-site coordination may be ignored at room temperature. In contrast, when the solution is stirred, the mechanism of a $1: 2$ coordination may take part in the reaction. The measurements of temperature dependence yield the activation energies, which remain constant, no matter whether the solution is agitated or not. The activation energy for the two-site coordination is found to be more difficult to surmount by a factor of 10 .

Acknowledgment. This work is supported by the National Science Council of the Republic of China under Contract No. NSC 93-2113-M-002-028.

\section{References and Notes}

(1) Bren, V. A.; Dubonosov, A. D.; Minkin, V. I.; Chernoivanov, V. A. Russ. Chem. Rev. 1991, 60, 451.

(2) Bren, V. A.; Dubonosov, A. D.; Minkin, V. I.; Chernoivanov, V. A. Russ. Chem. Rev. 2002, 71, 917.

(3) Bonfantini, E. E.; Officer D. L. J. Chem. Soc., Chem. Commun. 1994, 12, 1445

(4) Winkler, T.; Dix, I.; Jones, P. G., Herges, R. Angew. Chem., Int. Ed. 2003, 42, 3541.

(5) Watanabe, T.; Magaya, Y.; Yamashita, T.; Horie, K.; Nishikubo, T.; Morino, S. J. Photopolym. Sci. Technol. 1994, 7, 121.

(6) Herges, R.; Reif, W. Liebigs Ann. 1996, 5, 761.

(7) An, X.; Xie, Y. Thermochim. Acta 1993, 220, 17.

(8) Li, Z.; Scott, L. A. J. Phys. Chem. A 1998, 102, 9202.

(9) Hammond, G. S.; Turro, N J.; Fischer, A. J. Am. Chem. Soc. 1961, $83,4674$.

(10) Dilling, W. L. Chem. Lett. 1966, 66, 373.

(11) Miki, S.; Asako, Y.; Yoshida, Z. Tetrahedron Lett. 1988, 29, 2211.

(12) Hautala, R. R.; King, R. B.; Kutal, C. Solar Energy: Chemical Conversion and Storage; Humana Press: Clifton, NJ, 1979.

(13) Fife, D. J.; Morse, K. W.; Moore, W. M. J. Am. Chem. Soc. 1983, 105,7404 . 592.

(14) Ford, J. F.; Mann, C. K.; Vickers, T. J. Appl. Spectrosc. 1994, 48,

(15) Chuang, E. C. C.; Lin, K. C. J. Phys. Chem. B 2002, 106, 132.

9364.

(17) Patrick, T. B.; Bechtold, D. S. J. Org. Chem. 1984, 49, 1935.

(18) Noyori, R.; Umeda, I.; Kawauchi, H.; Takaya, H. J. Am. Chem. Soc. 1975, 97, 812 .

(19) Taylor, R. B.; Jennings, P. W. Inorg. Chem. 1981, 20, 3997.

(20) Sen, A.; Thomas, R. R. Organometallics 1982, 1, 1251.

(21) Lindberg, W.; Persson, J.-A. Anal. Chem. 1983, 55, 643.

(22) Phelan, M. K.; Barlow, C. H.; Kelly, J. J.; Jinguji, T. M.; Callis, J. B. Anal. Chem. 1989, 61, 1419.

(23) Schrieve, G. D.; Ullman, A. H. Appl. Spectrosc. 1991, 45, 713.

(24) Wang, C. C.; Chin, T. L.; Lin, K. C. J. Chem. Phys. 1997, 107, 10348

(25) Chin, T. L.; Lin, K. C. Appl. Spectrosc. 1999, 53, 22.

(26) Wang, C. C.; Chen Y. P.; Chin, T. L.; Lin, K. C. J. Chem. Phys. 2000, 112, 10204 . 